\title{
Optimal Bidding and Generation Scheduling of Utilities Participating in Single Side Auction Markets Including Ancillary Services
}

\author{
B. Rampriya \\ Department of Electrical and Computer Engineering, College of Technology, Debre Markos University, Ethiopia
}

\section{Article Info}

Article history:

Received May 23, 2017

Revised Nov 20, 2017

Accepted Jan 27, 2018

\section{Keywords:}

Differential Evolution (DE)

Generation Companies

(GENCOs)

Independent System Operator (ISO)

Market Clearing Price (MCP)

Quadratic Programming(QP)

\begin{abstract}
This paper presents the solution for the supplier's profit maximization problem with unit commitment decisions participating in single side auction markets of a deregulated power system. The bids from market participants are received by a central pool mechanism and the Market Clearing Price (MCP) for energy and spinning reserve is fixed. The bid quantities are optimized using Differential Evolution (DE) algorithm. The supplier aims to achieve (more) profit than that of the rival's participating in the competition. A GENCO with 6-unit participating in 24-hour day ahead energy and spinning reserve market is used to illustrate the methodology. The bidding parameters of rival's participating in the competition are calculated by multivariant Probability Density Functions (PDF). The results of the proposed methodology are compared with Refined Genetic Algorithm (RGA). Numerical results illustrate the effectiveness of the method in solving the supplier profit maximization problem.
\end{abstract}

Copyright () 2018 Institute of Advanced Engineering and Science. All rights reserved.

\section{Corresponding Author:}

B. Rampriya,

Department of Electrical and Computer Engineering,

College of Technology,

Debre Markos University, Ethiopia.

Email: rampriyame@gmail.com

\section{INTRODUCTION}

Ancillary services are those functions performed to support the basic services of generating capacity, energy supply and power delivery. Ancillary services are required for the reliable operation of the power system [1]. The general approach for pricing ancillary services within competitive electricity markets is based on fixed contracts for a certain time period between the ISO and the market participants that are able to provide the required ancillary services. Thereby, the ancillary services are divided into different services such as spinning reserves, non-spinning reserves, and Automatic Generation Control (AGC), replacement reserves, voltage support, and black start. The first four services can be procured by the ISO by means of daily competitive auction, whereas the last two services are more suitable for purchases based on long-term contracts [2]. In deregulated markets, there are separate auctions for each category of reserves. In this paper, it is assumed that GENCOs participate in the energy markets as well as in the ancillary service (only spinning reserve) auction markets.

The importance of spinning reserve which is required for system reliability is focused in this paper. Spinning reserve is the ability of an on-line generator (load) to increase (decrease) its output (consumption) in a short period of time. The time period will be determined by the system but for smaller systems the time period is generally smaller in order to avoid large frequency deviations [3].

Suppliers (GENCOs) and consumers (DISCOs) participate in the bidding process of double side auction markets in order to maximize the profit of suppliers and benefits of the consumers. This is achieved by differential evolution and dealt only the energy markets and not considered the reserve markets [4]. 
A method of building an optimal bidding strategy under market price uncertainty using information gap decision theory (IGDT) has been presented. A single thermal unit participating in day ahead energy markets without considering the reserve markets are addressed [5]. The supplier profit maximization problem is solved as multi objective optimization problem by considering the rival bidding and profit functions also by using Genetic Algorithm (GA) [6]. The supplier (decision maker) optimization problem is formulated under single side auction energy markets (without considering the spinning reserve markets) and their bid quantities are optimized using Self adaptive Differential Evolution (SaDE) [7].

In all the above literature mentioned, considerations have been made only to set up the energy markets and not on reserve markets. In this paper, an approach for providing this ancillary service is to set up reserve markets which run sequentially following the energy and transmission congestion management markets is dealt with. In this context, DE is employed to choose a GENCO's optimal bidding strategy among the sets of discrete bids.

This paper is organized as follows: Section 2 presents the market clearing mechanism in competitive energy and reserve markets, section 3 problem statement of profit maximization of decision makers submitting bids to market operators, section 4 deals with the solution methodology to find the optimum schedules of the supplier, section 5 presents the results and discussions, and section 6 concludes.

\section{ENERGY AND SPINNING RESERVE MARKETS}

In the restructured power systems, GENCOs will submit bid curves to the ISO, and then ISO clears market after collecting bids. In the ISO's market clearing model, ISO dispatches generating units in order from lowest to highest bid as needed to meet demand while considering network constraints. Once the energy market is cleared, each generating unit will be paid according to pricing mechanism of market. Generally there are two pricing mechanisms: pay-as-bid and uniform pricing [8]. Under the pay-as-bid pricing structure, every winning generating unit gets its bid price as its income. Under the uniform pricing structure, the bid price of the last dispatched unit sets the market clearing price, then all units dispatched receive the same MCP. In this paper, the uniform pricing structure is utilized.The formation and operation of energy markets were discussed in [7]. Spinning reserve, a generation based ancillary service can be made competitive and different from energy market. The spinning reserve service can be procured by ISO through daily competitive auctions. The $i$-th supplier spinning reserve bidding function can be represented as [9]

$$
\begin{aligned}
& S_{i}^{(t)}\left(R_{i}^{(t)}\right)=\varphi_{i}^{(t)}+\phi_{j}^{(t)} R_{i}^{(t)} t=1,2, \ldots \mathrm{T} \\
& \sum_{\mathrm{j}=1}^{N} R_{i}^{(t)}=\mathrm{SR}_{t} ; \mathrm{t}=1,2, \ldots \mathrm{T} \\
& R_{i \min } \leq R_{\mathrm{it}} \leq R_{i \max }
\end{aligned}
$$

where $S R_{t}$ is the reserve at hour $t, \varphi_{i}^{(t)}, \phi_{i}^{(t)}$ are the intercept and slope of the spinning reserve bidding curve of the suppliers respectively, $R_{\mathrm{it}}$ is the reserve generation output within the set of reserve limits $R_{i \min }$ and $R_{i \max }$. The MCP for spinning reserve (MCPR) is calculated as

$$
t=1,2, \ldots T
$$

The spinning reserve dispatch by each supplier can be calculated as

$$
R_{\mathrm{it}}=\left(\mathrm{MCPR}_{t}-\varphi_{i}^{(t)}\right) / \phi_{i}^{(t)} i=1,2 \ldots N
$$

\section{SUPPLIER PROFIT MAXIMIZATION PROBLEM FORMULATION}

The profit maximization objective of suppliers participating in energy and reserve markets and competing with the other suppliers can be stated as

Maximize: $P F=R V-T C$.

Profit $(P F)$ is defined as the revenue $(R V)$ from the sales of energy and reserve minus the total (production) cost $(T C)$.

$$
\mathrm{PF}=\sum_{\mathrm{i}=1}^{N} \sum_{\mathrm{t}=1}^{T}\left[\mathrm{MCP}_{t} \cdot P_{\mathrm{it}}+\mathrm{MCPR}_{t} \cdot R_{\mathrm{it}}-C_{j}\left(P_{\mathrm{it}}+\mathrm{R}_{\mathrm{it}}\right)\right] X_{\mathrm{it}}
$$


Where Xit the ON/OFF status of the suppliers decided by ISO and Ci(Pit) is the fuel cost function of the suppliers.

The constraints included are a) Power balance and b) Minimum and maximum capacity limits of suppliers.

a) Power balance constraints

The total generation (including spinning reserve) of GENCOs participating in the electricity markets may be greater than or equal to the demand profile of the customers.

$$
\sum_{\mathrm{j}=1}^{N}\left(P_{\mathrm{it}}+\mathrm{R}_{\mathrm{it}}\right) X_{\mathrm{it}} \geq \sum_{\mathrm{t}=1}^{T} D_{t} \mathrm{t}=1,2, \ldots T
$$

b) Minimum and maximum capacity limit constraints

Generation units have lower and upper production limits that are directly related to the generator design. These bounds can be defined as a pair of inequality constraints

$$
\begin{aligned}
& P_{i \min } \leq P_{\mathrm{it}} \leq P_{i \max } \\
& R_{i \min } \leq R_{\mathrm{it}} \leq R_{i \max } \\
& P_{\mathrm{it}}+\mathrm{R}_{\mathrm{it}} \leq P_{i \max }
\end{aligned}
$$

\section{SOLUTION METHODOLOGY}

The solution methodology of the decision maker profit maximization with optimized strategy problem is given as follows:

1. Initialization and creation of parent population: Set iteration count as 1 . One of the bidding parameter of the suppliers in energy markets $\left(\beta_{i}\right)$ and reserve markets $\left(\varphi_{i}\right)$ are optimized using a suitable algorithm. Here DE is employed. The bidding coefficient of suppliers in energy markets $\left(\alpha_{i}\right)$ and reserve markets $\left(\varphi_{i}\right)$ is kept fixed as the cost coefficients $b_{i}$ and $0.5 b_{i}$ respectively.

2. Calculation of bidding coefficients of rivals': The bidding parameters of the rivals' can be determined by statistical approach as given below.

The bid coefficients of rivals participating in energy markets, $\beta \mathrm{i}$ and $\alpha \mathrm{i}(\mathrm{i}=1,2 \ldots \mathrm{N})$ obey a multi-variate normal distribution with the PDF given in [10] and can be expressed in compressed form as

$$
\left(\alpha_{i}^{(t)}, \beta_{i}^{(t)}\right) \sim N\left\{\left[\begin{array}{l}
\mu_{\mathrm{i}, \mathrm{t}}^{(\alpha)} \\
\mu_{\mathrm{i}, \mathrm{t}}^{(\beta)}
\end{array}\right],\left[\begin{array}{cc}
\left(\sigma_{\mathrm{i}, \mathrm{t}}^{(\alpha)}\right)^{2} & \rho_{\mathrm{i}, \mathrm{t}} \sigma_{\mathrm{i}, \mathrm{t}}^{(\alpha)} \sigma_{\mathrm{i}, \mathrm{t}}^{(\beta)} \\
\rho_{\mathrm{i}, \mathrm{t}} \sigma_{\mathrm{i}, \mathrm{t}}^{(\alpha)} \sigma_{\mathrm{i}, \mathrm{t}}^{(\beta)} & \left(\sigma_{\mathrm{i}, \mathrm{t}}^{(\beta)}\right)^{2}
\end{array}\right]\right\}
$$

where $\rho_{\mathrm{i}, \mathrm{t}}$ is the correlation coefficient between $\alpha_{i}^{(t)}$ and $\beta_{i}^{(t)}, \mu_{\mathrm{i}, \mathrm{t}}^{(\alpha)}, \mu_{\mathrm{i}, \mathrm{t}}^{(\beta)}, \sigma_{\mathrm{i}, \mathrm{t}}^{(\alpha)}$ and $\sigma_{\mathrm{i}, \mathrm{t}}^{(\beta)}$ are the parameters of the multi-variant normal distribution.

The bid values of rival suppliers in energy markets are estimated as

$$
\begin{gathered}
\mu_{\mathrm{i}, \mathrm{t}}^{(\alpha)}=1.2 b_{i}, \mu_{\mathrm{i}, \mathrm{t}}^{(\beta)}=1.2 \times 2 a_{i} \\
4 \sigma_{\mathrm{i}, \mathrm{t}}^{(\alpha)}=0.15 b_{i}, 4 \sigma_{\mathrm{i}, \mathrm{t}}^{(\beta)}=0.15 a_{i} \\
\rho_{\mathrm{i}, \mathrm{t}}=-0.1
\end{gathered}
$$

The rivals' are expected to bid 20\% above operating cost. The mean and standard deviation of $\alpha_{i}$ and $\beta_{i}$ are specified as $\left[\mu_{\mathrm{i}, \mathrm{t}}^{(\alpha)}-4 \sigma_{\mathrm{i}, \mathrm{t}}^{(\alpha)}, \mu_{\mathrm{i}, \mathrm{t}}^{(\alpha)}+4 \sigma_{\mathrm{i}, \mathrm{t}}^{(\alpha)}\right]$ and $\left[\mu_{\mathrm{i}, \mathrm{t}}^{(\beta)}-4 \sigma_{\mathrm{i}, \mathrm{t}}^{(\beta)}, \mu_{\mathrm{i}, \mathrm{t}}^{(\beta)}+4 \sigma_{\mathrm{i}, \mathrm{t}}^{(\beta)}\right]$ respectively, with the probability of 0.999.

Similarly the bid coefficient of the rivals participating in spinning reserve markets, $\varphi_{i}$ and $\phi_{i}$ can be determined as given below. 


$$
\begin{gathered}
\mu_{\mathrm{i}, \mathrm{t}}^{(\varphi)}=0.5 \mu_{\mathrm{i}, \mathrm{t}}^{(\alpha)}, \mu_{\mathrm{j}, \mathrm{t}}^{(\phi)}=0.5 \mu_{\mathrm{i}, \mathrm{t}}^{(\beta)} \\
\sigma_{\mathrm{i}, \mathrm{t}}^{(\varphi)}=0.5 \sigma_{\mathrm{i}, \mathrm{t}}^{(\alpha)}, \sigma_{\mathrm{j}, \mathrm{t}}^{(\phi)}=0.5 \sigma_{\mathrm{i}, \mathrm{t}}^{(\beta)} \\
\gamma_{\mathrm{i}, \mathrm{t}}=\rho_{\mathrm{i}, \mathrm{t}}
\end{gathered}
$$

It is assumed that the rival bidding coefficients are same for all the 24 hours. It is not the case in practical situations. But in real time, the subsequent hour bids are estimated using the previous hour bidding data.

3. Calculation of MCP and MCPR: The MCP and MCPR are calculated with the bidding data of suppliers and rivals'. Based on the market price, $P_{\mathrm{it}}$ and $R_{\mathrm{it}}$ are calculated and limit values are checked.

4. Determination of unit ON/OFF status: If $P_{\mathrm{it}}+\mathrm{R}_{\mathrm{it}}<\mathrm{P}_{i \min }$, then $X_{\mathrm{it}}=0 \mathrm{else} X_{\mathrm{it}}=1$. Thus the unit ON/OFF Xit status can be calculated by taking an account of the constraints to be satisfied in all trading periods.

5. Economic Dispatch: With the calculated Xit, the optimal dispatch of power Pit and spinning reserve power Rit are calculated using Quadratic Programming (QP). The revenue generated and fuel costs spent are determined.

6. Calculation of fitness: The fitness is calculated as per equation (6).

7. Stopping criteria: The steps from 1 to 6 are repeated until the specified maximum number of iterations is reached.

\section{RESULTS AND DISCUSSION}

To illustrate the optimal bidding strategy, a GENCO with six suppliers are considered to be participating in 24 hour day-ahead electricity market. The results of test systems with and without optimized bidding strategies are tabulated. The generator and load data of the test system are taken from [11] and given in Appendix as Table A1 and A2 respectively. The proposed methodology is implemented on INTEL core, i3 processor, 3GB RAM and simulated in MATLAB 7.10 (R2010a) environment.The working algorithm used here is the seventh strategy of DE i.e. DE/rand/1/bin in which DE represents differential evolution, rand is any randomly chosen vector for perturbations, 1 represents the number of difference vectors to be perturbed and bin is the binomial type of crossover used. The seventh strategy is the most successful and widely used in optimization problems such as emission constrained economic dispatch [12], optimal power flow [13] and optimal design of gas transmission network [14].

\subsection{Parameter Selection}

The results are sensitive to algorithm parameters. Hence, it is required to perform repeated simulations to find the suitable values for the parameters. Optimal parameter combinations are experimentally determined by conducting experiments with different parameter settings. The following control parameters have been chosen for the test system.

Population size $N P=250$

Crossover Ratio $C R=0.9$

Differentiation or mutation constant $F=0.5$

Maximum number of iterations, MAXITER $=200$.

\subsection{Optimal Bidding Strategy Using DE}

The supplier-6 aims to maximize its own profit and other generators (1 to 5 ) are its rivals'. This example system is utilized for 24 hour (load) demand. The spinning reserve is maintained as $10 \%$ of the demand. The fuel cost equation is expressed in quadratic form as

$$
C_{i}\left(P_{\mathrm{it}}+\mathrm{R}_{\mathrm{it}}\right)=\mathrm{a}_{i}\left(P_{\mathrm{it}}+\mathrm{R}_{\mathrm{it}}\right)^{2}+\mathrm{b}_{i}\left(P_{\mathrm{it}}+\mathrm{R}_{\mathrm{it}}\right)+\mathrm{c}_{i}
$$

The supplier who is aware of market power in deregulated market is likely to bid above the marginal production cost. Hence the optimum values of $\beta_{6}$ and $\varphi_{6}$ are searched using DE within the intervals [1.05 $\times 2 \mathrm{a}_{6}$, $\left.1.35 \times 2 \mathrm{a}_{6}\right]$ and $0.5 \times\left[1.05 \times 2 \mathrm{a}_{6}, 1.35 \times 2 \mathrm{a}_{6}\right]$ respectively. The algorithm used here is the seventh strategy of DE i.e. $\mathrm{DE} / \mathrm{rand} / 1 / \mathrm{bin}$.

Based on the optimized bidding value of supplier-6 obtained from DE technique and rivals' bidding value from PDF, MCP and MCPR are fixed by PX and ISO respectively. The power dispatch and spinning reserve allocation of the suppliers is calculated with MCP and MCPR values in all trading hours. If the supplier is not able to provide minimum power requirement, then the corresponding supplier is not allowed to participate in the competition. Thus the ON/OFF commitment of the suppliers determined by pool operators for all the 24 hours and the economic power dispatch in all the trading hours is calculated using QP. It is observed that in the first 3 hours and $5^{\text {th }}$ hour, sixth supplier is in OFF condition because of bidding scheme. Since in these trading hours, supplier-6 cannot be able to supply even minimum requirement. So, the 
supplier-6 is made OFF during these hours. Suppose if the pool operator allows supplier-6 to enter into competition, then economic loss may occur during these hours.

The commitment schedule and power dispatch in energy and spinning reserve markets of the suppliers are presented in Table 1. The values of MCP of energy and spinning reserve, revenue generated, cost spent obtained for all the 24 trading hours are tabulated in Table 2.

Table 1. Power Dispatch in Energy and Spinning Reserve Markets

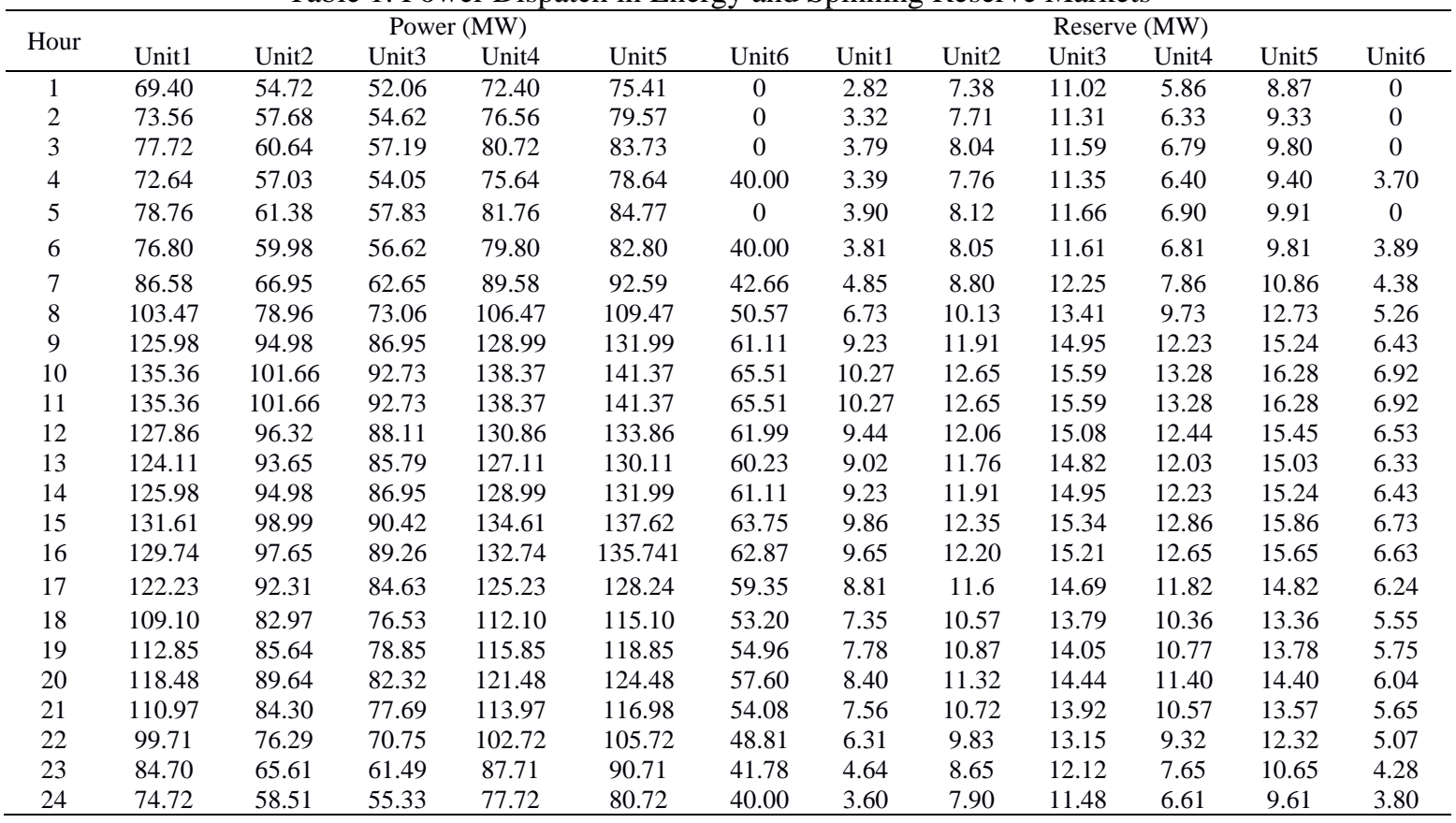

Table 2. Summary of Results of Supplier-6

\begin{tabular}{|c|c|c|c|c|c|c|}
\hline Hour & $\begin{array}{c}\text { Total power } \\
\text { generation(MW) }\end{array}$ & $\begin{array}{c}\text { Total reserve } \\
\text { generation(MW) }\end{array}$ & $\operatorname{MCP}(\$)$ & MCPR (\$) & Total Revenue(\$) & Total Cost (\$) \\
\hline 1 & 323.99 & 35.95 & 4.3207 & 1.0345 & 0 & 0 \\
\hline 2 & 341.99 & 38.00 & 4.4596 & 1.0414 & 0 & 0 \\
\hline 3 & 360.00 & 40.01 & 4.5984 & 1.0483 & 0 & 0 \\
\hline 4 & 378.00 & 42.00 & 4.7373 & 1.0551 & 193.39 & 191.36 \\
\hline 5 & 364.5 & 40.49 & 4.6332 & 1.0500 & 0 & 0 \\
\hline 6 & 396.00 & 43.98 & 4.8761 & 1.0620 & 199.18 & 192.19 \\
\hline 7 & 441.01 & 49.00 & 5.2233 & 1.0791 & 227.54 & 205.88 \\
\hline 8 & 522.00 & 57.99 & 5.8481 & 1.1099 & 301.56 & 246.99 \\
\hline 9 & 630.00 & 69.99 & 6.6812 & 1.1510 & 415.71 & 308.43 \\
\hline 10 & 675.00 & 74.99 & 7.0283 & 1.1681 & 468.48 & 336.25 \\
\hline 11 & 675.00 & 74.99 & 7.0283 & 1.1681 & 468.48 & 336.25 \\
\hline 12 & 639.00 & 71.00 & 6.7506 & 1.1544 & 426.01 & 313.89 \\
\hline 13 & 621.00 & 68.99 & 6.6118 & 1.1476 & 405.52 & 303.02 \\
\hline 14 & 630.00 & 69.99 & 6.6812 & 1.1510 & 415.71 & 308.43 \\
\hline 15 & 657.00 & 73.00 & 6.8895 & 1.1613 & 447.00 & 324.96 \\
\hline 16 & 648.00 & 71.99 & 6.8200 & 1.1579 & 436.45 & 319.40 \\
\hline 17 & 611.99 & 67.98 & 6.5423 & 1.1442 & 395.46 & 297.66 \\
\hline 18 & 549.00 & 60.98 & 6.0564 & 1.1202 & 328.44 & 261.65 \\
\hline 19 & 567.00 & 63.00 & 6.1952 & 1.1270 & 346.97 & 271.67 \\
\hline 20 & 594.00 & 66 & 6.4035 & 1.1373 & 375.70 & 287.11 \\
\hline 21 & 557.99 & 61.99 & 6.1258 & 1.1236 & 337.653 & 266.63 \\
\hline 22 & 504.00 & 56 & 5.7092 & 1.1031 & 284.25 & 237.49 \\
\hline 23 & 432.00 & 47.99 & 5.1538 & 1.0757 & 219.93 & 201.57 \\
\hline 24 & 387.00 & 43 & 4.8067 & 1.0585 & 196.288 & 191.77 \\
\hline \multicolumn{7}{|c|}{ Total Profit / day = \$1487.1 } \\
\hline
\end{tabular}

Figure 1. shows the variation of MCP and MCPR with respect to trading hours from 1 to 24 . The MCP is fixed based on the load demand profile. For the given load profile, MCP is increasing from 1 to 11. 
At $12^{\text {th }}$ hour, load demand is decreased and thus there is reduction of energy and reserve price. It is observed that the energy price is about four times higher than reserve price.

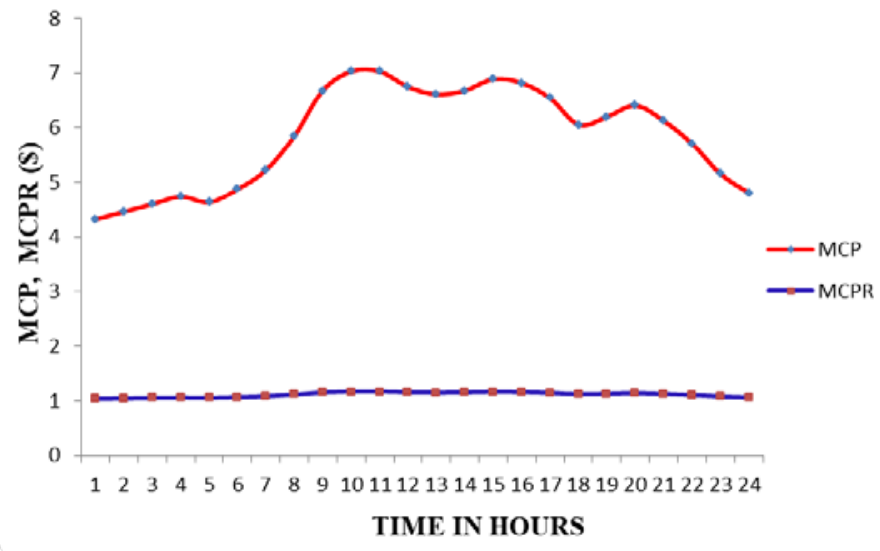

Figure 1. Variations of MCP and MCPR

The performance and effectiveness of the proposed methodology is examined in comparison to the solutions given by RGA [11]. Table 3 shows the comparison of hourly profit of the proposed DE with optimized bids and RGA methods.

Table 3 Comparison of Hourly Profit by DE and RGA Methods

\begin{tabular}{|c|c|c|c|c|c|}
\hline \multirow{2}{*}{ Hour } & \multicolumn{2}{|c|}{ Profit (\$) } & \multirow{2}{*}{ Hour } & \multicolumn{2}{|c|}{ Profit (\$) } \\
\hline & RGA [11] & $\mathrm{DE}$ & & RGA [11] & $\mathrm{DE}$ \\
\hline 1 & 0 & 0 & 13 & 93.78 & 102.5028 \\
\hline 2 & 0 & 0 & 14 & 97.94 & 107.2823 \\
\hline 3 & 0 & 0 & 15 & 110.8 & 122.0424 \\
\hline 4 & 2.92 & 2.0362 & 16 & 106.5 & 117.0521 \\
\hline 5 & 0 & 0 & 17 & 89.67 & 97.7936 \\
\hline 6 & 8.22 & 6.9913 & 18 & 62.51 & 66.7957 \\
\hline 7 & 22.47 & 21.6651 & 19 & 69.98 & 75.3010 \\
\hline 8 & 51.73 & 54.5647 & 20 & 81.62 & 88.5858 \\
\hline 9 & 97.94 & 107.2823 & 21 & 66.22 & 71.0132 \\
\hline 10 & 119.6 & 132.2337 & 22 & 44.83 & 46.7619 \\
\hline 11 & 119.6 & 132.2337 & 23 & 19.51 & 18.3609 \\
\hline 12 & 102.1 & 112.1321 & 24 & 5.54 & 4.5137 \\
\hline \multicolumn{4}{|c|}{ Total Profit (\$) } & $\begin{array}{c}\text { RGA } \\
\text { DE }\end{array}$ & $\begin{array}{c}1373.48 \\
14871\end{array}$ \\
\hline
\end{tabular}

It is clear that the supplier-6 receives high profit in all the trading hours when it submits the bids by optimizing the bid coefficients using DE to ISO. The profit distribution of supplier-6 is high in all the trading hours and can be seen in Figure 2. There is a net profit difference of $\$ 113.62$ for supplier-6 between RGA and DE.

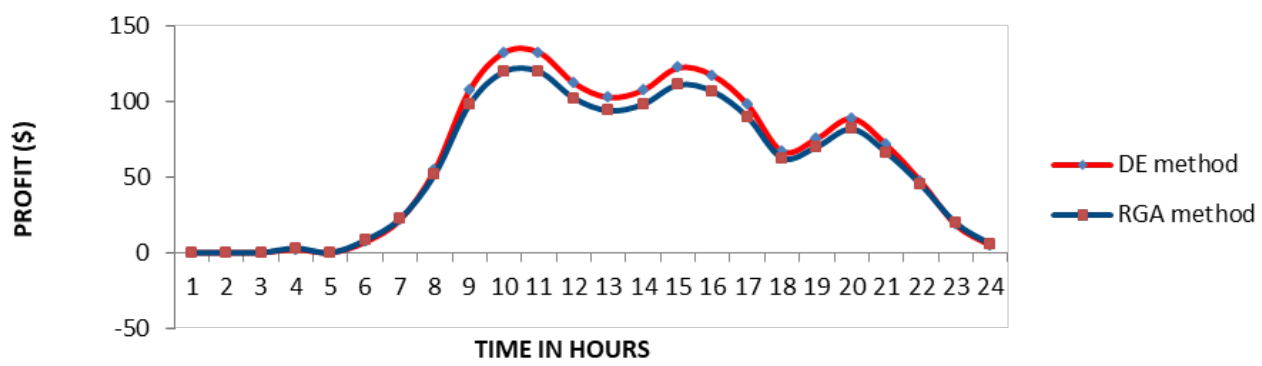

Figure 2. Comparison of Hourly Profit of Supplier-6 
The convergence characteristic of the system is shown in Figure 3. The maximum number of iterations is fixed at 200 and the algorithm is able to converge before 150 iterations in all the runs. The best solutions found for the problem are tabulated. The highest profit obtained in simulation using DE is \$1487.1. Out of 50 simulation runs, the proposed algorithm produces feasible solutions in 21 runs with best profit value of $\$ 1487.1$. The simulation results obtained in 50 independent runs using DE are given in Table 4. The best and worst profits obtained are \$1487.1and \$1382.5 respectively.

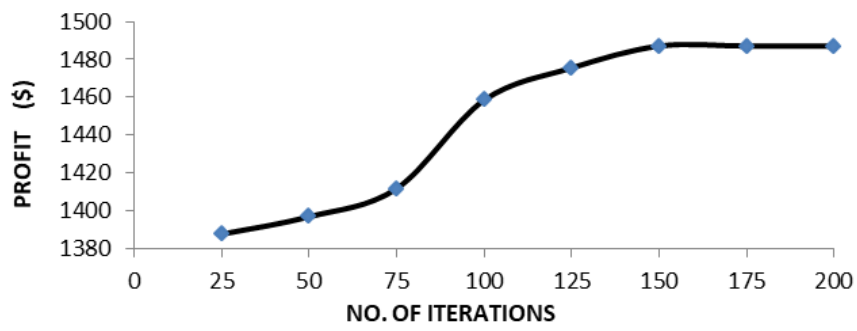

Figure 3. Convergence Characteristics

Table 4 Summary of Simulation Results for 50 Independent Trial Runs

\begin{tabular}{ccccccc}
\hline No. of trials & Revenue (\$) & Cost (\$) & Profit (\$) & $\begin{array}{c}\text { Mean value of } \\
\text { profit (\$) }\end{array}$ & SD of profit & $\begin{array}{c}\text { Best value of } \\
\text { profit (\$) }\end{array}$ \\
\hline 4 & 8668.2 & 7280.6 & 1387.6 & & \\
7 & 7602.9 & 6128.6 & 1474.4 & & \\
8 & 8707.6 & 7325.1 & 1382.5 & 1442.2 & 52.38 & 1487.1 \\
10 & 7468.2 & 5988.9 & 1479.3 & & \\
21 & 6889.721 & 5402.6 & 1487.1 & & & \\
\hline
\end{tabular}

Experimentation has also been carried out by calculating $\beta_{6}$ and $\varphi_{6}$ with joint PDF and without optimizing these bid coefficients. In this case, ISO fixes the price and arrives at a schedule in such a way that the supplier-6 is put OFF during hours 1 to 5. So, the total profit of supplier-6 in this case is reduced to $\$ 1369.30$. Table 5 shows the comparison of simulation results of optimal bidding strategy using RGA, DE and bidding values by joint PDF. The average execution time for a single run using DE is about 12.64 seconds. The main difference in constructing better solutions is that RGA relies on crossover while DE relies on mutation operation and thus DE algorithm faces a promising approach for solving supplier optimization problem.

Table 5 Comparison of Results of 6 Unit 24 Hour System

\begin{tabular}{ccc}
\hline Method & Profit (\$) & Number of trial runs \\
\hline RGA [11] & 1373.48 & -- \\
PDF (without bid optimization) & 1369.30 & -- \\
DE (with bid optimization) & 1487.1 & 21 \\
\hline
\end{tabular}

\section{CONCLUSION}

The methodology using DE is proposed to determine optimal bidding strategy for a GENCO in 24hour energy and reserve markets. GENCO submits 24 hourly supply-bidding curves for energy to the PX, and 24 hourly supply-bidding curves for reserve to the system operator. Based on bidder information, load demand and reserve, the energy and reserve awarded to each bidder are determined. The proposed method is developed based on the viewpoint of the GENCO as a supplier wishing to maximize profit. Investigation reveals that DE performs much better than GA in terms of convergence rate, quality of solution and success rate. The DE algorithm can solve the problem efficiently and accurately. 


\section{APPENDIX}

Table A1. Generator Data of Test System

\begin{tabular}{|c|c|c|c|c|c|}
\hline Unit & $\mathrm{a}_{\mathrm{i}}\left(\$ / \mathrm{MWh}^{2}\right)$ & $\mathrm{b}_{\mathrm{i}}(\$ / \mathrm{MWh})$ & $\mathrm{C}_{\mathrm{i}}(\$ / \mathrm{h})$ & $\mathrm{P}_{\min }(\mathrm{MW})$ & $\mathrm{P}_{\max }(\mathrm{MW})$ \\
\hline 1 & 0.0125 & 2 & 0 & 40 & 160 \\
\hline 2 & 0.0175 & 1.75 & 0 & 40 & 140 \\
\hline 3 & 0.02 & 1.5 & 0 & 30 & 120 \\
\hline 4 & 0.0125 & 1.9 & 0 & 40 & 170 \\
\hline 5 & 0.0125 & 1.8 & 0 & 40 & 180 \\
\hline 6 & 0.0275 & 1.85 & 58 & 40 & 100 \\
\hline
\end{tabular}

Table A2. Load Data of Test System

\begin{tabular}{|c|c|c|c|c|c|c|c|c|c|c|c|c|}
\hline Hour & 1 & 2 & 3 & 4 & 5 & 6 & 7 & 8 & 9 & 10 & 11 & 12 \\
\hline Load(MW) & 360 & 380 & 400 & 420 & 405 & 440 & 490 & 580 & 700 & 750 & 750 & 710 \\
\hline Hour & 13 & 14 & 15 & 16 & 17 & 18 & 19 & 20 & 21 & 22 & 23 & 24 \\
\hline Load(MW) & 690 & 700 & 730 & 720 & 680 & 610 & 630 & 660 & 620 & 560 & 480 & 430 \\
\hline
\end{tabular}

\section{REFERENCES}

[1] Gibescu. M and Liu C.C, "Optimization of ancillary services for system security”, Proceedings of Bulk power system dynamics and control IV- Restructuring, symposiums, pp. 351-358, 1998.

[2] Singh. H and A. Papalexopoulos, "Competitive procurement of ancillary services by an independent system operator”, IEEE Trans Power Systems, Vol.14, No.2, pp. 498-504, 1999.

[3] Sullivan M. J and Malley M. J, "A new methodology for the provision of reserve in an isolated power system", IEEE Transactions on Power Systems, Vol.14, pp. 174-183, 1999.

[4] Angatha, V.V.S., Chandram, K. and Laxmi, A.J, "Bidding Strategy in Deregulated Power Market Using Differential Evolution Algorithm”, Journal of Power and Energy Engineering, 3, 37-46, 2015.

[5] Sayyad Nojavan, Kazem Zare, Mohammed Reza, “Optimal bidding strategy of generation station in power market using Information gap decision theory”, Electric Power Systems Research, 96, 26-63, 2013.

[6] Azedeh. A, Ghaderi.S.F, Pourvalikhan. B, Sheikhalishahi. M, "A new genetic algorithm approach for optimizing bidding strategy view point of profit maximization of a generation company”, Expert systems with applications, 39, 1565-1574, 2012.

[7] B. Rampriya, "Profit maximization and optimal bidding strategies of GENCOs in electicity markets using self adaptive differential evolution”, International Journal of Electrical Engineering and Informatics, Vol. 8, No. 4, 2016.

[8] Soleymani. S, "Bidding strategy of generation companies using PSO combined with SA method in the pay as bid markets”, Electrical Power and Energy Systems, Vol. 33, pp. 1272-1278, 2011.

[9] Fushuan Wen and A. K. David, "Strategic bidding in reserve market", Proceedings of the 5th International Conference on Advances in Power System Control, Operation and Management, APSCOM 2000, Hong Kong, pp. 80-85, 2000.

[10] Fushuan Wen, A. Kumar David, "Optimal bidding strategies and modeling of imperfect information among competitive generators”, IEEE Transactions on power systems, Vol. 16, No.1, pp. 15-21, 2001.

[11] Fushuan Wen and A.K. David, "Coordination of bidding strategies in day-ahead energy and spinning reserve markets”, International Journal of Electrical Power and Energy Systems, Vol. 24, pp. 251-261, 2002.

[12] Abou A. A, Abido. M.A, Spea S.R, "Differential Evolution algorithm for emission constrained economic power dispatch problem”, Electric Power Systems Research, Vol. 80, pp.1286-1292, 2010.

[13] Abou El ElaA.A., M.A. Abido, S.R. Spea, “Optimal power flow using differential evolution algorithm”, Electric Power Systems Research, Vol. 80, pp. 878-885, 2010.

[14] Babu B.V, P.G. Chakole, J.H.S. Mubeen, "Differential Evolution Strategy for Optimal Design of Gas Transmission Network”, available online at: www.vsppub.com, 2010. 